\title{
Variation of modal parameters of a highway bridge extracted from six earthquake records
}

\author{
Hugo C. Gomez, Hasan S. Ulusoy* ${ }^{* \dagger}$ and Maria Q. Feng \\ Department of Civil and Environmental Engineering, University of California, Irvine, CA 92697, USA
}

\begin{abstract}
SUMMARY
Between 2005 and 2010 six earthquakes triggered a monitoring system consisting of 11 acceleration channels installed on the West Street On-Ramp, a three-span curved highway bridge located in the city of Anaheim, California. In this paper, three different system identification techniques are applied to the acceleration records to investigate and corroborate the dynamic properties of the bridge, that is, vibration frequencies, associated damping ratios and mode shapes. The identification techniques are applied to each one of the six seismic events. The identified frequencies and damping ratios are shown to be dependent variables of the earthquake intensity. In general, larger earthquake intensities result in reduced vibration frequencies and higher damping ratios of the bridge. Sensitivity analysis using a simple finite element model reveals that soil softening at the abutments considerably contributes to the variation in frequencies because of changes in the support conditions and ultimately in the global stiffness of the structure. In addition, mathematical models in the state space description are identified from the recorded response and excitation measurements. The state space models successfully replicate the bridge measured response to the earthquake from which it is constituted. The models also provide a reasonable prediction of the bridge response to a different earthquake. Copyright (C) 2012 John Wiley \& Sons, Ltd.
\end{abstract}

Received 4 February 2011; Revised 1 June 2012; Accepted 5 June 2012

KEY WORDS: modal parameters; the state space model; seismic response; system identification; highway bridge

\section{INTRODUCTION}

After being applied for the first time in control design, system identification (SI) has gained enormous popularity in the field of structural engineering over the last three decades. From the structural engineering point of view, SI refers to the estimation of dynamic properties of structures based on measured response to different sorts of excitations such as ground motion, traffic, wind, and wave. In this paper the application of SI techniques to problems of earthquake and bridge engineering is presented. Because earthquakes are one of the primary sources that may cause damage to structures, it is necessary to study their dynamic behavior on the basis of the measured data including both excitation (input) and response (output) for a better understanding of their dynamic behavior during earthquakes. Through the application of these techniques, important insights into the dynamic behavior of structures during earthquakes can be obtained. This will allow engineers to revise modeling and analysis procedures, and ultimately to improve design codes.

Earthquake response data have been used to identify mathematical models of civil engineering structures and their vibration characteristics afterwards [1-3]. The input-output model was adopted in those studies where the response of structures is considered as the output, and the ground motion

*Correspondence to: Hasan S. Ulusoy, Department of Civil and Environmental Engineering, University of California, Irvine, CA 92697, USA.

†E-mail: hulusoy@uci.edu 
is considered as the input. Considering the recordings of certain channels on structures as the input leads to the use of the transmissibility functions. In general, the transmissibility functions cannot be treated as the frequency response functions (FRFs), thus they do not provide the modal parameters of the structure. However, application of the transmissibility functions where a structure is excited at its supports is a special case (base excitation). In such a case, taking the support motion as excitation, the transmissibility functions can be treated as the FRFs and the natural frequencies, damping ratios, and unscaled mode shapes of the structure can be extracted. More details on the use of the transmissibility functions in modal testing can be found in [4].

Traditionally, ground motion is considered as excitation in the identification of structural systems. There are however cases where it is impractical to measure excitation. The term 'output-only' refers to such cases where excitation measurement is not available, and response measurement is the only data to be utilized in identification procedure. The assumption that excitation is a white noise process leads to the development of SI techniques suitable for output-only systems. A review on SI techniques for output-only systems is given in [5]. It is also shown that several techniques developed for output-only systems have their input-output counterparts. For cases where both excitation and response are available, SISO, and MIMO models are used to describe the dynamics of a structural system. MIMO models are used more frequently when both excitation and response are measured at several locations on a structure.

The time-domain SI techniques have been successfully applied to study the dynamic behavior of bridge structures on the basis of the measured response data during earthquakes. For example, the frequencies and damping ratios of the two dominant modes of the San Juan Bautista Bridge are estimated using the first seismic response recorded for a bridge in California [6]. Another example of the application of a system identification technique to the earthquake response data of the Meloland Road Overpass, a well-instrumented highway bridge, is reported in [7]. In that paper, the first few vibration modes of the bridge that participate significantly in response are identified using the bridge's response to the 1979 Imperial Valley earthquake. It is also observed that the transverse response of the bridge is controlled by the abutment motions. Later, the performance of the two finite element models (a linear stick model and a detailed nonlinear model) of the Painter Street Overpass is compared [8]. This study concludes that the response of the bridge superstructure is considerably influenced by the behavior of the abutments and approach embankment soil. In another publication the frequencies and damping ratios of the first vibration modes of a curved highway bridge are estimated from the 1992 Landers and Big Bear earthquake records using parametric and nonparametric SI techniques [9]. An increase in the period of the fundamental vibration mode is found from one earthquake to another. Later, both weak and strong ground motions are used to assess seismic response characteristics of a five-span continuous bridge [10]. That study shows that weak and strong ground excitation induce significant differences on the dynamic response of the bridge. Soon after, the modal parameters of the seven highway bridges in California during different earthquakes are identified using Auto-Regressive model with eXogenous input (ARX) and a nonparametric technique [11]. Then, a comparison of the performance of three MIMO techniques including various configurations of sensors and different earthquake records is presented in [12]. The comparison shows slightly different natural frequencies during each earthquake.

Despite the important contributions to the identification of bridge structures many uncertainties remain on how earthquake intensity affects the identification of bridge modal parameters. In this paper, system identification of the West Street On-Ramp (WSOR) bridge using six earthquake records of different intensities is presented. There are three motivating factors for this study: (i) to investigate the effect of earthquake intensity on identified bridge modal parameters; (ii) to study factors influencing the change in the identified bridge modal parameters, including the change of support conditions; and (iii) to present simple state space models generated using the SI results to replicate the measured response and to predict the bridge's response to different inputs. Therefore, the objectives of the paper are as follows:

i. To show the practical use of recorded earthquake data for identification of modal parameters of a highway bridge using three different SI techniques;

ii. To examine the effect of earthquake intensity on the identified modal parameters;

iii. To replicate and predict the bridge response to earthquake loads using the state space models generated from the SI results. 
This paper studies the dynamic behavior of the WSOR bridge on the basis of the measured response data during five earthquakes. Three MIMO SI techniques are employed to identify the dynamic properties of the bridge. A comparison of SI results using the six different earthquake data sets is then presented to investigate the relationship between earthquake intensity and identified modal parameters. An examination of how changing boundary conditions affect the bridge natural frequencies is presented based on a sensitivity analysis by varying the lateral and rotational spring stiffness values associated with the abutments in a simple finite element model of the bridge. Finally, the authors consider that the identified modal parameters can be used in future works for a better understanding of the seismic response of curved RC bridges.

\section{DESCRIPTION AND INSTRUMENTATION OF THE BRIDGE}

The WSOR is a $151.3 \mathrm{~m}(496.5 \mathrm{ft})$ long horizontally curved bridge located on the Santa Ana Freeway (I-5) in the city of Anaheim, California. The basic dimensions of the structure are shown in Figure 1. The WSOR is a three-span continuous bridge with a single-cell cast-in-place prestressed and posttensioned concrete box girder. The three span lengths are $45.8 \mathrm{~m}(150.2 \mathrm{ft}), 60.1 \mathrm{~m}(197.3 \mathrm{ft})$, and $45.4 \mathrm{~m}(149.1 \mathrm{ft})$ running from south to north. The super-elevation is $12 \%$ and the vertical alignment is slightly curved. The radius of curvature is $167.6 \mathrm{~m}(550 \mathrm{ft})$. At the beginning and end of the bridge, the deck is resting on two reinforced elastomeric bearing pads at seat-type abutments. The two bents located at the ends of the middle span have a single circular column with a $2.7 \mathrm{~m}(9 \mathrm{ft})$ diameter. All substructure elements are cast-in-place concrete supported on steel pipe piles and oriented normal to the centerline of the bridge. The pier footing piles are steel pipes and have a $356 \mathrm{~mm}(14 \mathrm{in})$ diameter and about $10 \mathrm{~m}(33 \mathrm{ft})$ long. The deck supports two $822.9 \mathrm{~mm}(2.7 \mathrm{ft})$ high concrete barriers and a $1.6 \mathrm{~m}(5.3 \mathrm{ft})$ high sound wall traveling all along the bridge. Construction of the bridge was completed in 2001 and was designed under the American Association of State Highway and Transportation Officials 1983 Standard Specifications for Highway Bridges with interims and revisions by Caltrans [13].

The WSOR is the first curved bridge to be instrumented in Orange County, California. The sensor layout is shown in Figure 1. Along the center line of the bridge deck, five stations were instrumented with either a uniaxial (models SV-155-H, SV-156-V) or a biaxial (models SV-255-HH, SV-256-HV) force-balanced servo-type accelerometer. A total of eight channels simultaneously record the bridge response consisting of two vertical (up - \#'s 3,10), five transversal (across the bridge - \#'s $1,2,5,9,11$ ) and one longitudinal (along the bridge - \# 4) acceleration time-histories. In addition, a triaxial accelerometer was placed at the base of Bent 2, just above the top elevation of the footing,
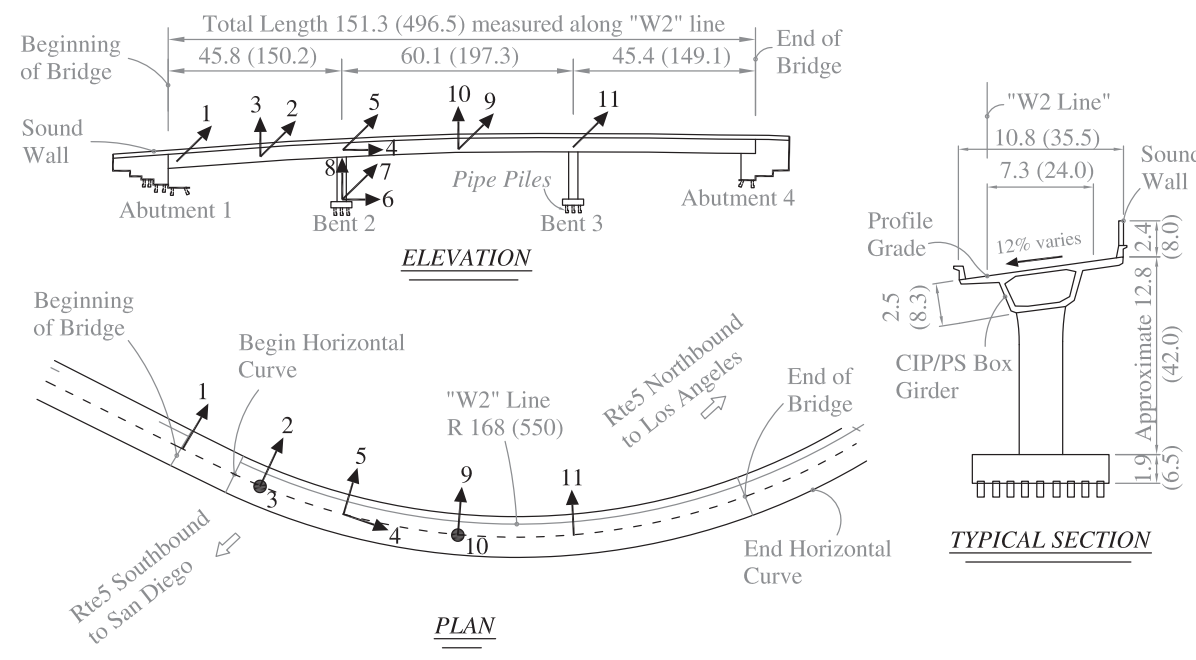

Figure 1. The West Street On-Ramp basic dimensions and sensor layout. Dimensions in meters (ft). 
to record inputs (ground acceleration) consisting of three channels (\#'s 6,7,8) in the longitudinal, transversal and vertical directions, respectively. The recorded data is sampled at $100 \mathrm{sps}$. The resolution of the accelerometers is $0.0001 \%$ of full range maximum and the Analog-to-Digital converter resolution of the data recorder is 22 bits. The monitoring system is triggered when an acceleration larger than or equal to $0.002 \mathrm{~g}$ is registered at either channel 6,7 or 8 . Once the monitoring system is triggered all the accelerometers start recording at the same time.

\section{SEISMIC ACCELERATION RECORDS}

From 2005 to 2010 the monitoring system at the WSOR bridge site has recorded six earthquakes. These earthquakes are listed in Table I together with some relevant information. The earthquake data are composed of low and moderate ground motion intensities at the base of Bent 2. The weakest event, the Calexico earthquake, occurred on April 04, 2010 with a PGA of $0.007 g$ and the strongest one, the Chino Hills earthquake, occurred on July 29, 2008 with a PGA of $0.367 g$ recorded both at channel 7.

Figure 2 shows the collection of ground motion records at the base of Bent 2 of the six earthquakes. All have been plotted to the same acceleration and time scale. For all events the PGA in transverse direction is nearly an order of magnitude larger than in the longitudinal direction whereas the PGA in the vertical direction is the smallest among the three components. The free-field measurements are not available so the recording at the base of Bent 2 are considered to be the input acceleration, which excites the bridge structure.

Table I. Earthquake events recorded at the bridge site.

\begin{tabular}{lcccccr}
\hline & & & & & \multicolumn{2}{c}{ PGA $(g)$} \\
\cline { 5 - 7 } Event & Date & Magnitude & Distance $(\mathrm{km})$ & Longitudinal & Transverse & Vertical \\
\hline Anza & June 12, 2005 & 5.2 & 129 & 0.005 & 0.011 & 0.002 \\
Yucaipa & June 16, 2005 & 4.9 & 88 & 0.006 & 0.018 & 0.005 \\
Chino Hills & July 29, 2008 & 5.5 & 21 & 0.086 & 0.367 & 0.045 \\
Inglewood & May 17, 2009 & 4.7 & 41 & 0.013 & 0.026 & 0.007 \\
Pico Rivera & March 16, 2010 & 4.4 & 24 & 0.010 & 0.019 & 0.004 \\
Calexico & April 4, 2010 & 7.2 & 300 & 0.006 & 0.007 & 0.004 \\
\hline
\end{tabular}

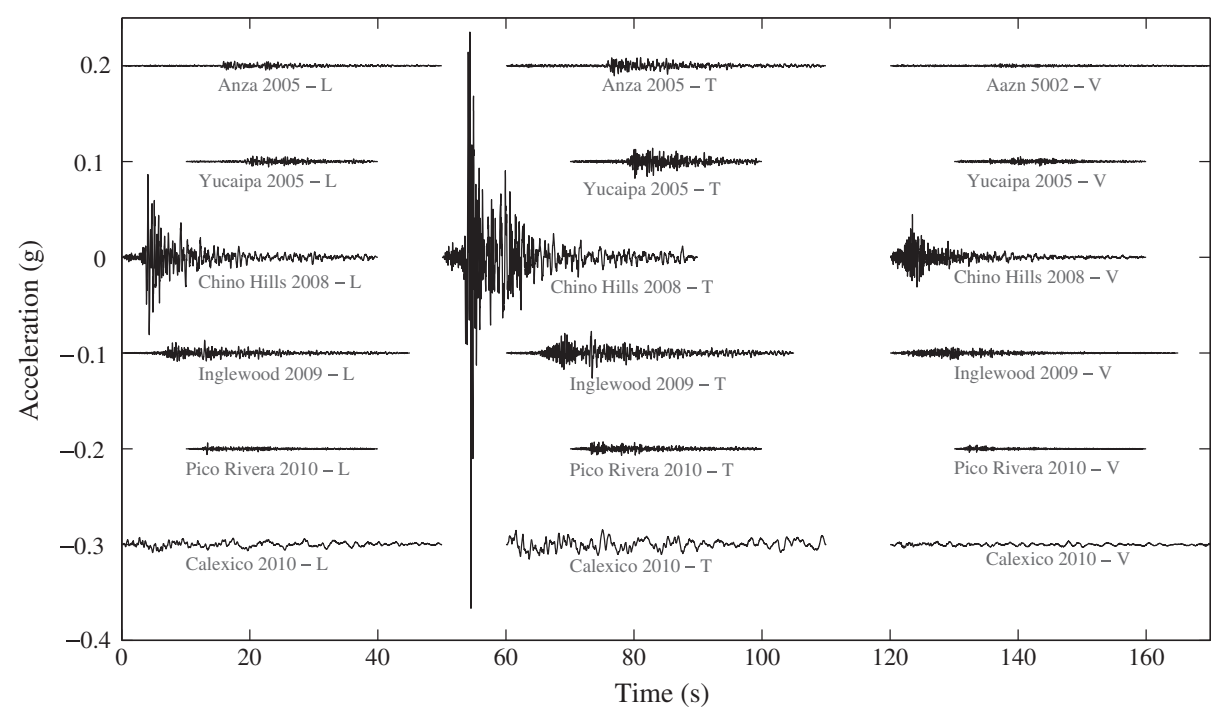

Figure 2. Ground motion time-histories at the base of Bent 2 recorded on WSOR during six earthquakes. 
All events were successfully recorded except for the Calexico earthquake, which was longer than the 1-min maximum capture length of the data recorder. This fact was corroborated by correlating acceleration-time records from the Center for Engineering Strong Motion Data at different stations with the records at the bridge site. However, this is not an impediment for system identification purposes; thus, all six earthquake data were analyzed in this paper to facilitate a comparison of the estimated modal properties of the WSOR.

The Fourier spectra for all the records are shown in Figure 3. The frequency content for all earthquakes is rich enough to excite the bridge lower modes of vibration, which are usually in the interval from 0.7 to $5 \mathrm{~Hz}$ for concrete short-span bridges [14, 15]. Only the Calexico earthquake shows low frequency contents after $2 \mathrm{~Hz}$ most likely because of dissipation through long distance wave propagation causing higher frequency components to damp out.

\section{SYSTEM IDENTIFICATION TECHNIQUES}

Three time-domain system identification techniques are briefly described in this section. The techniques utilize the measured input and output data to build the mathematical model of a system. First, a model structure is adopted. Then, the parameters of the model are estimated using the measured data. Once the model is identified, the dynamic properties of the system can be readily extracted from the model.

\subsection{Subspace system identification techniques}

A finite dimensional, linear, time-invariant system can be described in the discrete time-domain by the state space model as

$$
\begin{aligned}
\mathbf{x}(k+1) & =\mathbf{A} \mathbf{x}(k)+\mathbf{B u}(k)+\mathbf{w}(k) \\
\mathbf{y}(k) & =\mathbf{C x}(k)+\mathbf{D u}(k)+\mathbf{v}(k)
\end{aligned}
$$

where $\mathbf{x} \in \mathbb{R}^{n}$ is the state vector, $\mathbf{y} \in \mathbb{R}^{l}$ is the measurement vector, $\mathbf{u} \in \mathbb{R}^{m}$ is the excitation vector, while $\mathbf{A} \in \mathbb{R}^{n \times n}$ is the state matrix, $\mathbf{B} \in \mathbb{R}^{n \times m}$ is the input matrix, $\mathbf{C} \in \mathbb{R}^{l \times n}$ is the output matrix, $\mathbf{D} \in \mathbb{R}^{l \times m}$ is the direct transmission matrix, and $k$ is the discrete time variable. The modeling
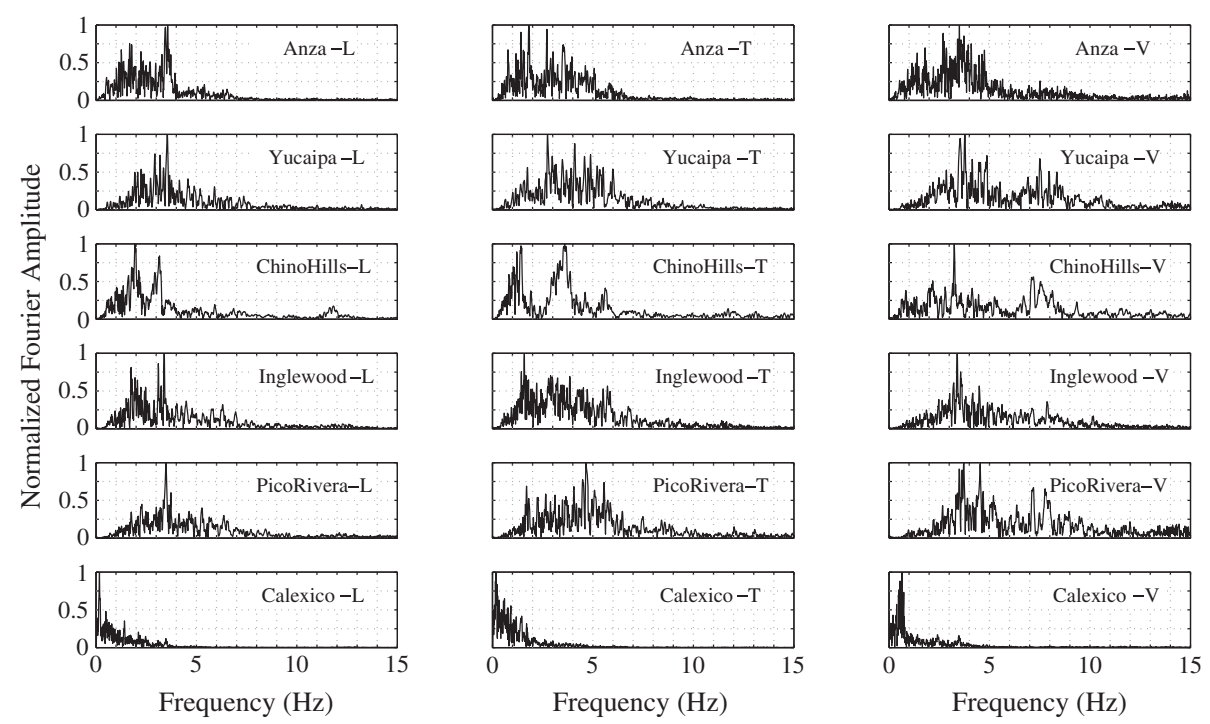

Figure 3. Fourier spectra of the six ground motions. 
uncertainties and the measurement errors are represented by zero mean white noise processes $\mathbf{w}$ and $\mathbf{v}$, respectively. In subspace techniques, the system matrices $(\mathbf{A}, \mathbf{B}, \mathbf{C}, \mathbf{D})$ are estimated in different ways. First, the extended observability matrix and system order are directly determined from input-output data using geometrical tools such as orthogonal projection, oblique projection, or canonical correlation. The system matrices $(\mathbf{A}, \mathbf{C})$ can be then extracted from the extended observability matrix. Once the system matrices $(\mathbf{A}, \mathbf{C})$ are estimated, the input-output description of the system becomes linear in matrices $\mathbf{B}$ and $\mathbf{D}$, thus they can be readily estimated by the least squares method. The subspace state space system identification, multivariable output error state sPace (MOESP) model identification, and canonical variate analysis are the three well-known subspace techniques in the literature [16-18]. In the MOESP technique, one goes back to the input-output data again, while the processed data and/or the estimated states after the $\mathrm{QR}$ and singular value decomposition are used to estimate matrices (B,D) in the subspace state space system identification technique. Similarities and differences between subspace techniques are discussed in [19, 20].

\subsection{Eigensystem realization algorithm with observer (Kalman filter) identification}

The Eigensystem realization algorithm (ERA) is a time-domain technique utilized to extract modal parameters from the impulse response function of a system [21]. For the case when the impulse response is not measured directly, the ERA was later extended by adding a procedure, called Observer (Kalman filter) IDentification, to recover the impulse response from the input-output data [22]. The impulse response is then stored in a block Hankel matrix. The singular value decomposition of the Hankel matrix provides the extended observability and controllability matrices. The system matrices $(\mathbf{A}, \mathbf{B}, \mathbf{C})$ can be extracted from the extended observability and controllability matrices. The ERA originates from the Ho-Kalman realization algorithm [23]. The ERA with Observer (Kalman filter) IDentification procedure provides a unified approach to build state space models of linear time-invariant systems from the measured input-output data. The applications of the technique to civil engineering structures using earthquake response data were reported in [1-3].

\subsection{Autoregressive model with exogenous input}

Autoregressive models have been often used in statistics and economics for prediction and identification. The most simple input-output relationship of a linear system can be described by an ARX as follows:

$$
\mathbf{y}(k)+\sum_{j=1}^{n_{a}} \mathbf{A}_{j} \mathbf{y}(k-j)=\sum_{j=1}^{n_{b}} \mathbf{B}_{j} \mathbf{u}\left(k+1-n_{d}-j\right)+\mathbf{e}(k)
$$

where $\mathbf{u} \in \mathbb{R}^{m}$ and $\mathbf{y} \in \mathbb{R}^{l}$ are the input and output measurement vectors, respectively, $\mathbf{e} \in \mathbb{R}^{l}$ is a zero mean white noise disturbance, the matrices, $\mathbf{A}_{j} \in \mathbb{R}^{l \times l}, j=1, \ldots, n_{a}$, are autoregressive model coefficients and the matrices, $\mathbf{B}_{j} \in \mathbb{R}^{l \times m}, j=1, \ldots, n_{b}$, account for the influence of the past input on the output, while $\left(n_{a}, n_{b}\right)$ and $n_{d}$ are the model order and delay parameters, respectively. The model orders relate the number of the system poles, the number of zeros, and the response delay. Given the input and output measurements, the determination of the matrix coefficients of the ARX model becomes a linear regression problem, thus the solution for the unknown coefficients can be obtained by the least squares method. The orders of the ARX model are first determined and the unknown coefficients are estimated afterwards. In general, an ARX model of higher orders $\left(n_{a}, n_{b}\right)$ fits the measured data better than those of lower orders. The determination of the model orders is a trade-off process between the model complexity and the fit. The ARX model is used to study the effects of environmental factors and damage events on the variation in natural frequencies of a highway bridge [24].

\section{MODAL PARAMETER IDENTIFICATION OF THE WSOR BRIDGE}

It is often accepted that preprocessing of the data involving trend removal, filtering and decimation leads to more accurate identification results. Because all the SI techniques applied herein are capable 
of minimizing spurious results, even in the presence of noise, in this study only trend removal was applied to the raw data. For the WSOR, the transverse (across the bridge) and vertical responses are noticeably larger than the longitudinal response. Also, there are six transverse channels, which constitute the majority of the measurements. Therefore, the resulting configuration for SI is such that the inputs are channel \#'s 7, 8 and the outputs are channel \#'s 1, 2, 3, 5, 9, 10, and 11 .

\subsection{Natural frequencies}

Stabilization diagrams were used to help in picking the natural frequencies. Stabilization diagram is a tool used for discriminating spurious modes from the structural (physical) modes of vibration. This diagram shows frequencies as the horizontal axis and model order as the vertical axis. The poles (modes) corresponding to a certain model order are compared with the poles of a one-order-lower model. Physical poles (modes) are identified at the same frequency at increasing model orders forming a vertical line of stable poles. A detailed definition of stabilization diagram can be found elsewhere [25]. As an example, Figure 4 shows the stabilization diagram for the Chino Hills earthquake obtained using the ERA technique. The first natural frequency clearly appears at $1.55 \mathrm{~Hz}$ and the second at $2.19 \mathrm{~Hz}$. The former stabilizes at higher model orders whereas the latter stabilizes at lower model orders (around 20). The third frequency at $2.87 \mathrm{~Hz}$ only stabilizes at low model orders (from 15 to 30) showing a poor contribution of this mode in the bridge response. Nevertheless, the FRF taking channel 8 as input and channel 3 as output, both vertical, shows a peak near the third frequency. Note that the FRFs in Figure 4 are not computed directly from the data but extracted from the state space model being identified.

Figure 5 shows an acceptable agreement between the three SI techniques in identifying the first three natural frequencies using the six seismic acceleration records. For comparison purposes, in Figure 5 the black bars represent the average frequencies identified from a long-term monitoring (2002, 2004 to 2010) of the bridge traffic induced acceleration response using an output-only frequency domain method [26]. The grey scale bars represent those frequencies identified from the seismic records using three different SI techniques. For the strongest earthquake (Chino Hills) the identified frequencies are nearly $20 \%$ smaller than for all other records. An interesting observation is that the frequencies before (2008) and after (2009) the Chino Hills earthquake practically have the same values, meaning the system clearly changes its properties during the earthquake. One possible explanation for the change of the bridge frequencies is the changing boundary conditions such as soil properties at footings and abutments. Similar phenomena on a 4-story reinforced concrete building were observed [1].

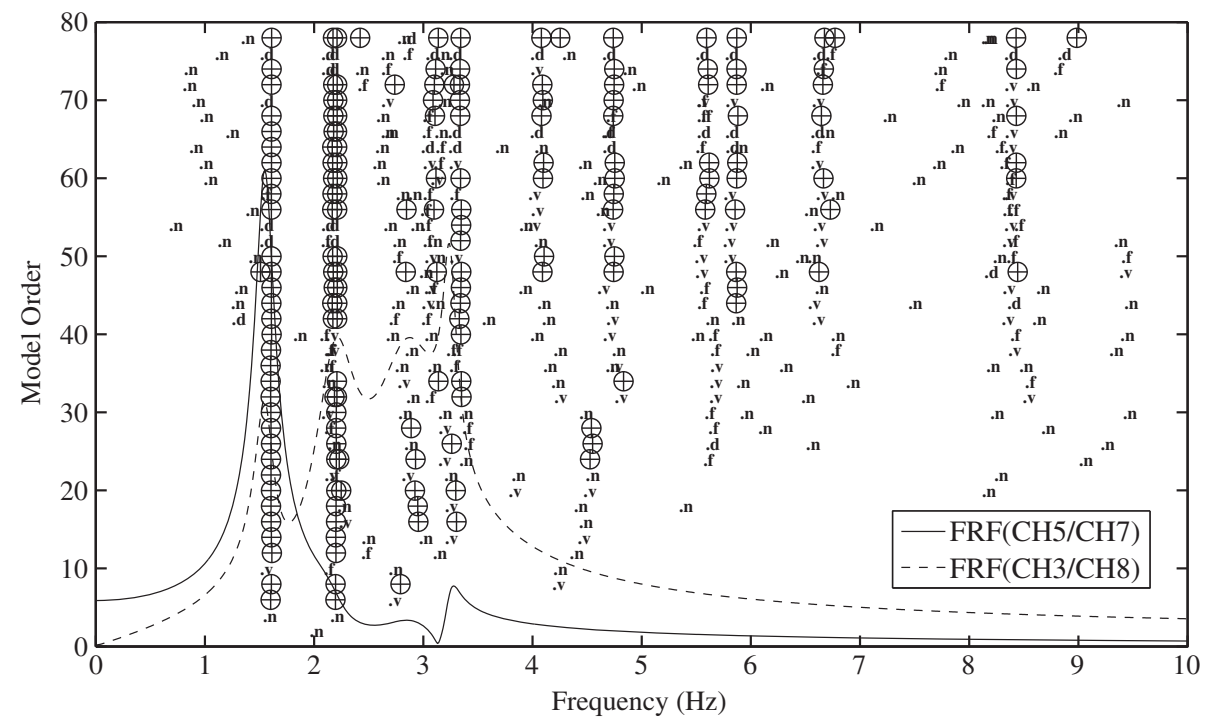

Figure 4. Stabilization diagram generated using acceleration records from Chino Hills earthquake and applying the ERA technique. The used symbols are: ' $\oplus$ ' for a stable pole; '. v' for a pole with stable frequency and vector; '.d' for a pole with stable frequency and damping; '.f' for a pole with stable frequency and '.n' for a new pole. 
1st Natural Frequency

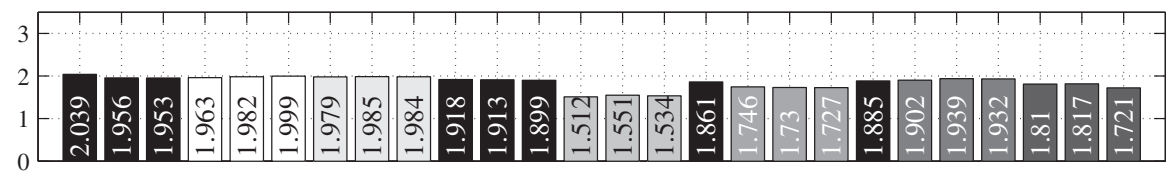

2nd Natural Frequency
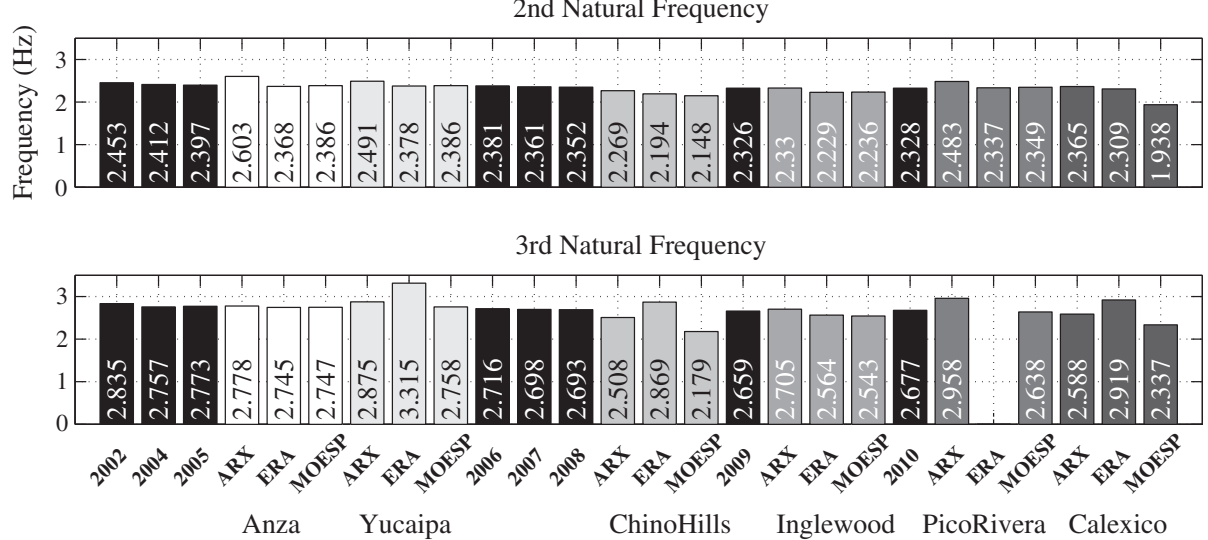

Figure 5. The first three natural frequencies of the WSOR bridge identified using six seismic records and traffic induced vibration records from 2002 to 2010.

The natural frequencies identified from the six earthquakes appear to decrease as the ground motion gets stronger (higher peak acceleration at the base of Bent 2), as shown in Figure 6, where the Chino Hills and the Calexico earthquakes are not included because of the significantly high peak acceleration of the former and the remoteness of the latter. The scattered points reflect only the results using the MOESP technique although the same trend is apparent for the three techniques. There is no sufficient diversity in the PGAs to clarify whether the trend approaches to a linear or a nonlinear relationship between the frequencies and the maximum acceleration at the base of the column.

5.1.1. Sensitivity of natural frequencies. As mentioned above a possible explanation for the abrupt change in the identified natural frequencies is the change in the bridge support conditions. Although the variability of modal parameters is often attributed to environmental effects, changes in traffic loads, and irregularities in modal testing procedures and data preprocessing [27], a significant reduction (nearly 20\%) of the first natural frequency of the WSOR bridge deserves special attention. Visual inspections of the WSOR carried out after the occurrence of the six earthquakes revealed the structural elements are in adequate conditions, thus the reduction in the frequency is attributed to a possible change in the support conditions during the earthquake rather than the presence of damage on the bridge. To examine how a change in boundary conditions and a change in stiffness affects the natural frequencies, a sensitivity analysis is carried out in this section.

Figure 7 shows a sensitivity analysis carried out using a simple finite element model of the WSOR bridge. The supports at both abutments were idealized as a linear spring assigned to each degree-of-freedom, three translational $\left(K_{x}, K_{y}, K_{z}\right)$ and three rotational $\left(K_{r x}, K_{r y}, K_{r z}\right)$, while the supports at the base of the columns were idealized as fixed. Here, $x, y$, and $z$ represent the longitudinal, transversal, and vertical axes, respectively. The sensitivity analysis consisted of varying the concrete Young's modulus for deck $\left(E_{\mathrm{d}}\right)$
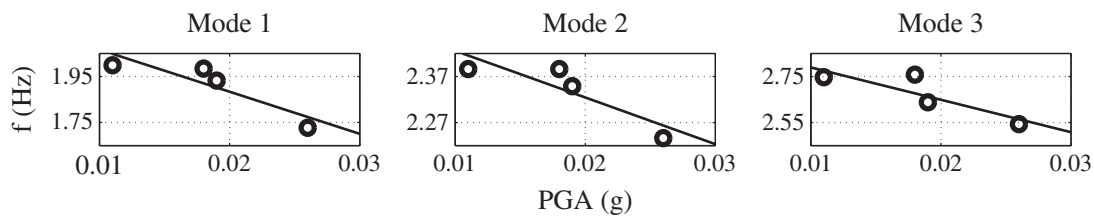

Figure 6. Line fit to natural frequencies of WSOR versus PGA at base of Bent 2. 


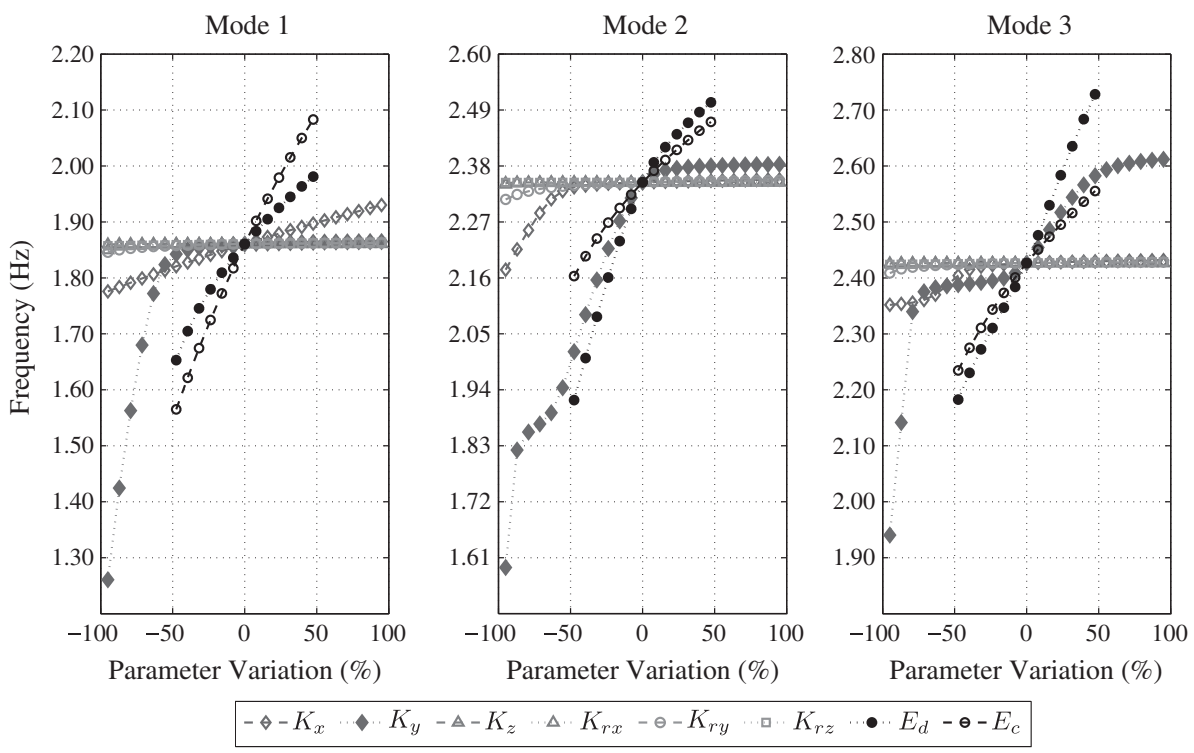

Figure 7. Sensitivity analysis of the finite element model of WSOR.

and columns $\left(E_{\mathrm{c}}\right)$ by $\pm 50 \%$ and the stiffness value assigned to each of the linear springs at the abutments by $\pm 100 \%$. Each parameter was analyzed one at a time. When the variation is set to zero this signifies the initial values are assigned to the model based on the design drawings. Because the estimation of Young's modulus initial values are based on the concrete compressive strength (determined using concrete cylinders at an age of 28 days), it can be concluded that the values do not vary significantly. On the other hand, estimation of the spring stiffness values at the abutments is not satisfactory because of uncertainties of the soil properties. Therefore, the spring stiffness values vary significantly in the sensitivity analysis, as shown in Figure 7.

The sensitivity analysis shows that the natural frequencies are more sensitive to the variation of the concrete Young's moduli of the deck and the columns than any of the other parameters. However, as mentioned above, the variation of Young's modulus cannot be significant. Therefore, it is the variation of the abutment spring stiffness in the transverse direction $\left(K_{\mathrm{y}}\right)$ that causes a significant reduction in natural frequencies. The sensitivity analysis implies that the supports at abutments may be loosened in the transverse direction during ground shaking. As the stiffness of the abutments in the transverse direction is reduced so is the natural frequency of the bridge. Therefore, the nearly $20 \%$ decrease of the first frequency is attributed to a change in the support conditions of the WSOR bridge.

\subsection{Damping ratios}

Figure 8 shows the identified damping ratios from the six ground motions. In this study, vibration modes were considered spurious and disregarded as structural modes when the identified modal damping ratios were above $20 \%$. In practice, it is difficult to estimate damping ratios accurately [28]. Commonly, it is expected that structures exhibit classically damped modes. As expected, for the WSOR the higher damping ratios correspond to the Chino Hills earthquake (5\% to $7 \%$ of the critical for the first mode). During the Chino Hills earthquake the energy dissipation at the bridge boundaries is thought to contribute to an increase in damping. In a previous study, researchers have concluded energy dissipation at a bridge's boundaries makes a significant contribution to the damping of the entire bridge system under strong motion [29]. For each frequency and damping ratio the relative error between the three system identification techniques (the ERA, the MOESP, and ARX) was computed. It was observed that the average relative error among the three SI techniques is of the order of 5\% for the frequencies, whereas for the damping estimates the average relative error is as high as $150 \%$. Thus, it can be sensibly argued that the estimates of the natural frequencies are more reliable than the estimates of the damping ratios. 
1st Modal Damping Ratio
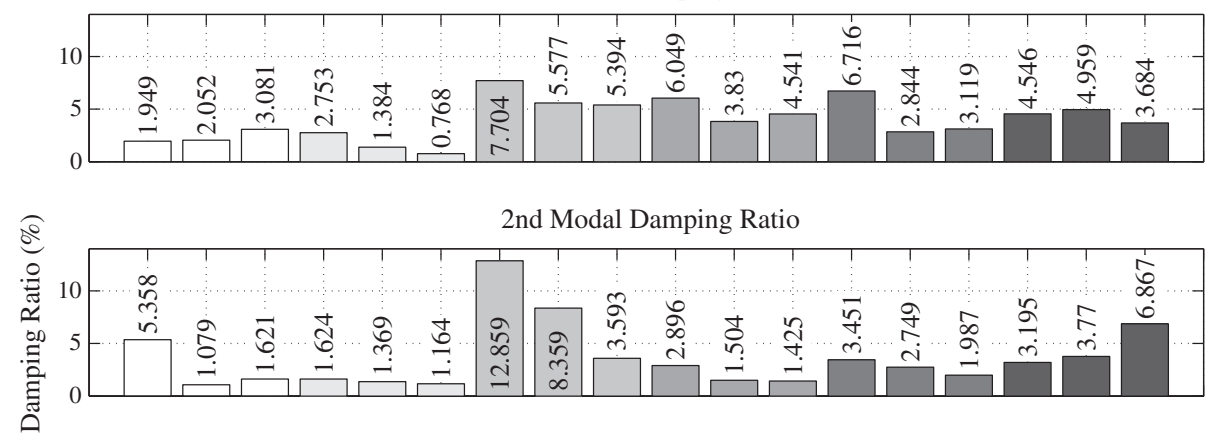

3rd Modal Damping Ratio

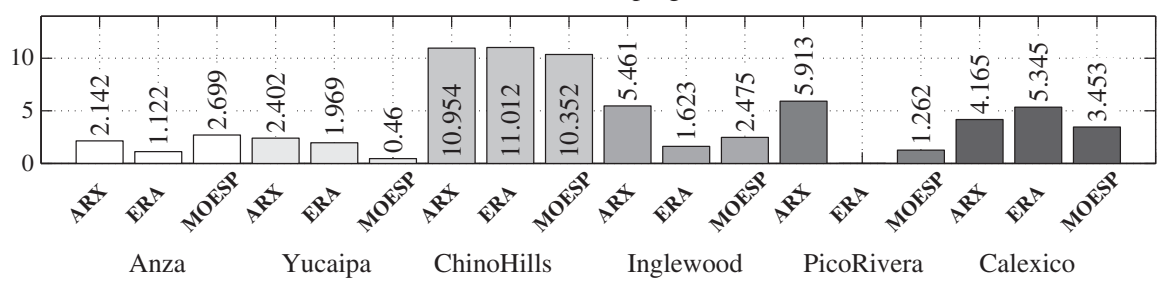

Figure 8. First three WSOR identified damping ratios using six seismic records.

\subsection{Mode shapes}

Mode shapes provide a global understanding of the dynamic behavior of the structure under different earthquake events. Because the applied SI techniques identify complex mode shapes, techniques should be adopted to estimate normal mode shapes from corresponding complex modes which are more general $[30,31]$. Nonetheless, if the real and the imaginary parts of a complex mode components are in phase or $180^{\circ}$ out of phase, the assumption of normal modes is valid and the system is approximated as a classically damped system [28]. In this study, an indicator of the complexity of mode shapes called modal phase colinearity was used to decide whether an identified mode can be considered as a normal mode. For the WSOR identified modes the modal phase colinearity was found to be above 0.90 for the first two modes and above 0.87 for the third mode. Therefore, the assumption of normal modes is fairly valid and the system can be treated as a classically damped system. Identified mode shapes of the WSOR bridge were compared using not only different earthquake events but also using different SI techniques. For this purpose, the consistency between two mode shapes being compared was measured using the modal assurance criterion (MAC)[32] given by,

$$
\operatorname{MAC}\left(\phi_{i}, \phi_{j}\right)=\frac{\left(\phi_{i}^{T} \cdot \phi_{j}\right)^{2}}{\left(\phi_{i}^{T} \cdot \phi_{i}\right)\left(\phi_{j}^{T} \cdot \phi_{j}\right)}
$$

The MAC can take on values from zero, meaning no consistency, to one, meaning consistency between the two modes. When comparing mode shapes associated to the first mode of vibration of the WSOR bridge, MAC values greater than 0.90 were obtained. Therefore, the first identified mode was consistent between the different earthquakes and SI techniques. Similarly, MAC values greater than 0.80 were obtained for the second mode of vibration. However, MAC values for the third mode shape were considerably lower (with an average of 0.5 ) than those obtained for the first and second modes. For example MAC values of $0.92,0.88$, and 0.34 for modes 1, 2, and 3, respectively, were obtained when one compares the mode shapes identified from the Yucaipa and Inglewood earthquakes using the MOESP and ARX techniques, respectively.

In general, the lowest MAC values were obtained for comparisons involving modes extracted from the Chino Hills earthquake data. One possible explanation is that the intensity of the Chino Hills earthquake is the greatest among all recorded seismic events, which caused the bridge system to 
change its dynamic characteristics. For instance, the Anza earthquake has a smaller intensity (PGA $=0.011 \mathrm{~g}$ ) than the Chino Hills earthquake $(\mathrm{PGA}=0.367 g$ ) resulting in that the obvious earthquake intensity affects not only the natural frequencies but also the modes of vibration. Moreover, previous studies have shown that at higher response levels, the bridge may deviate significantly from a lightly damped normal mode response [33].

Figure 9 shows 2D and 3D representations of the normalized mode shapes for the first three identified vibration modes using the Chino Hills earthquake. The identified space-discrete mode shapes in Figure 9 were interpolated between instrumented stations using cubic splines along the bridge deck. It is worth to mention that the three measured mode shapes have components in both transverse and vertical directions because of the curvature of the bridge. Even though the identified mode shapes combine horizontal and vertical displacements the first identified mode shape is primarily the bending of the deck in the $X Y$ plane (plan view), whereas the second mode is bending of the deck in the $X Z$ plane (elevation). The third mode is a combination of double curvature bending of the deck in transverse direction because of the out-of-phase movement of the columns plus vertical bending of the first span. An interesting observation is that the mode shape at channel \#1 (top of abutment 1) is nonzero, implying the abutments move in the transverse direction under seismic excitation. This can be seen especially for modes 1 and 3 inferring the boundary conditions can change during earthquakes leading to slightly different SI results.

\section{EARTHQUAKE RESPONSE PREDICTION BY THE STATE SPACE MODELS}

With the purpose of replicating the measured response time-history, which is assumed to be the 'truesystem-response', linear state space models of the WSOR were generated for each one of the six earthquakes. Subsequently, the models were individually tested to predict the response to a different earthquake event. Unlike finite element models, physical parameters, that is, mass, stiffness and damping matrices, cannot be estimated with any of the three SI techniques presented in this study. However, the generated first-order models of the bridge have the advantage to handle both the classical and the nonclassical damping cases equally well [3]. Although they are not extremely precise they are able to give insight of the bridge response to different earthquakes, leading to invaluable information for maintenance and future retrofit designs.

Because of space limitations, Figure 10 shows the replication of the WSOR response at channels 5 (transverse) and 10 (vertical) only for Yucaipa, Chino Hills and Inglewood earthquakes using the MOESP SI technique. The root mean squared error (RMSE) is included to compare the accuracy of the match between channels. The results show that the match for the vertical response has less accuracy than the match for the transverse response. One possible explanation is that the models do not consider such factors as vehicle loadings that are acting on the bridge deck in the vertical direction. Even though the probability of having a vehicle crossing the bridge during earthquake

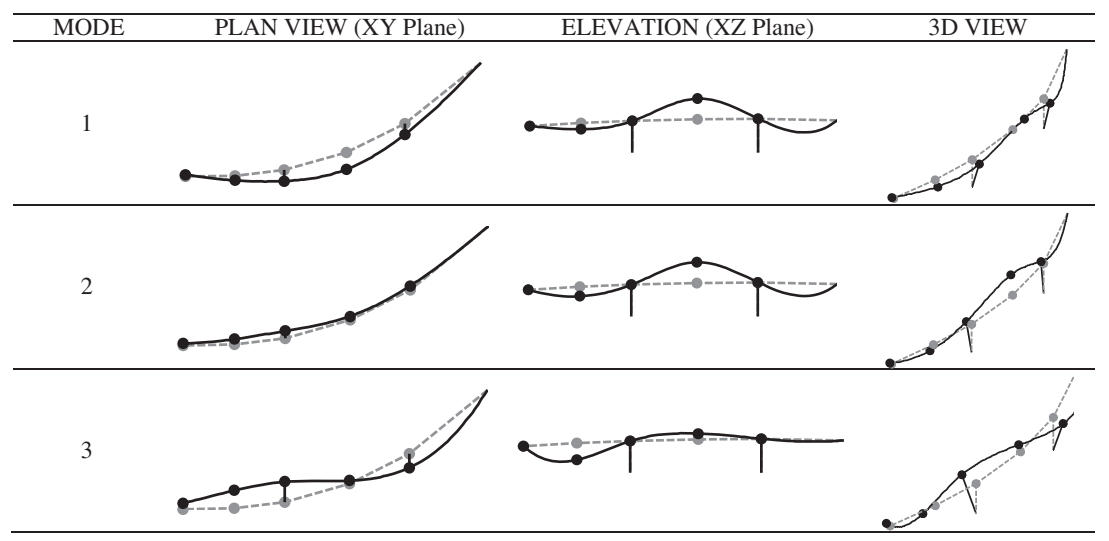

Figure 9. Two-dimensional and three-dimensional representation of the first three WSOR measured mode shapes. The solid circles represent the instrumented stations on the deck. 


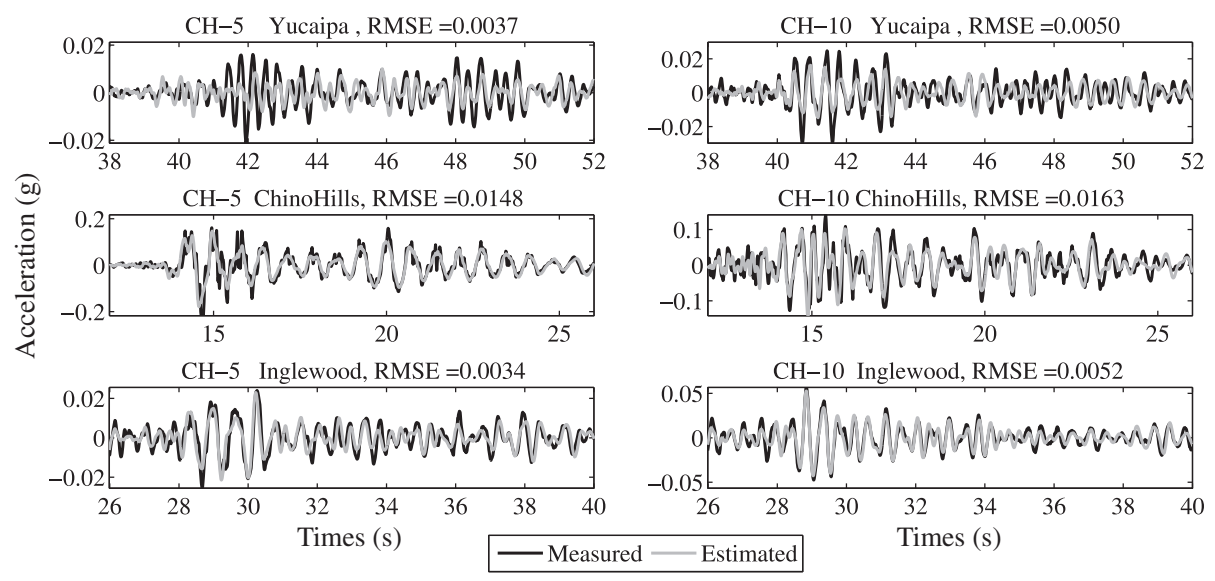

Figure 10. Measured and estimated bridge acceleration response at channels 5 (transverse) and 10 (vertical) using state space models constructed by the MOESP technique.

occurrence is usually disregarded, inertial forces in a highly curved bridge with pronounced camber, such as the WSOR, could lead to an increase in magnitude of the vertical response at midspans. The discrepancies are inevitable considering the models are reduced in order and do not consider the nonlinearity inherent to the system. Any gross mismatch indicates a deficiency so a refined analysis must be taken into consideration if an accurate match is pursued. In this study the match between measured and replicated responses is considered to have accuracy sufficient so the model mimics the bridge response. Hence, the estimated modal parameters are within desired bounds. Similar results were obtained using the ERA technique, however, RMSE values indicate that the MOESP technique gives slightly better results when estimating the bridge seismic response.

Each state space model was constructed using the MOESP technique and tested to predict the response to a different earthquake event. Figure 11 displays the predicted response to an earthquake
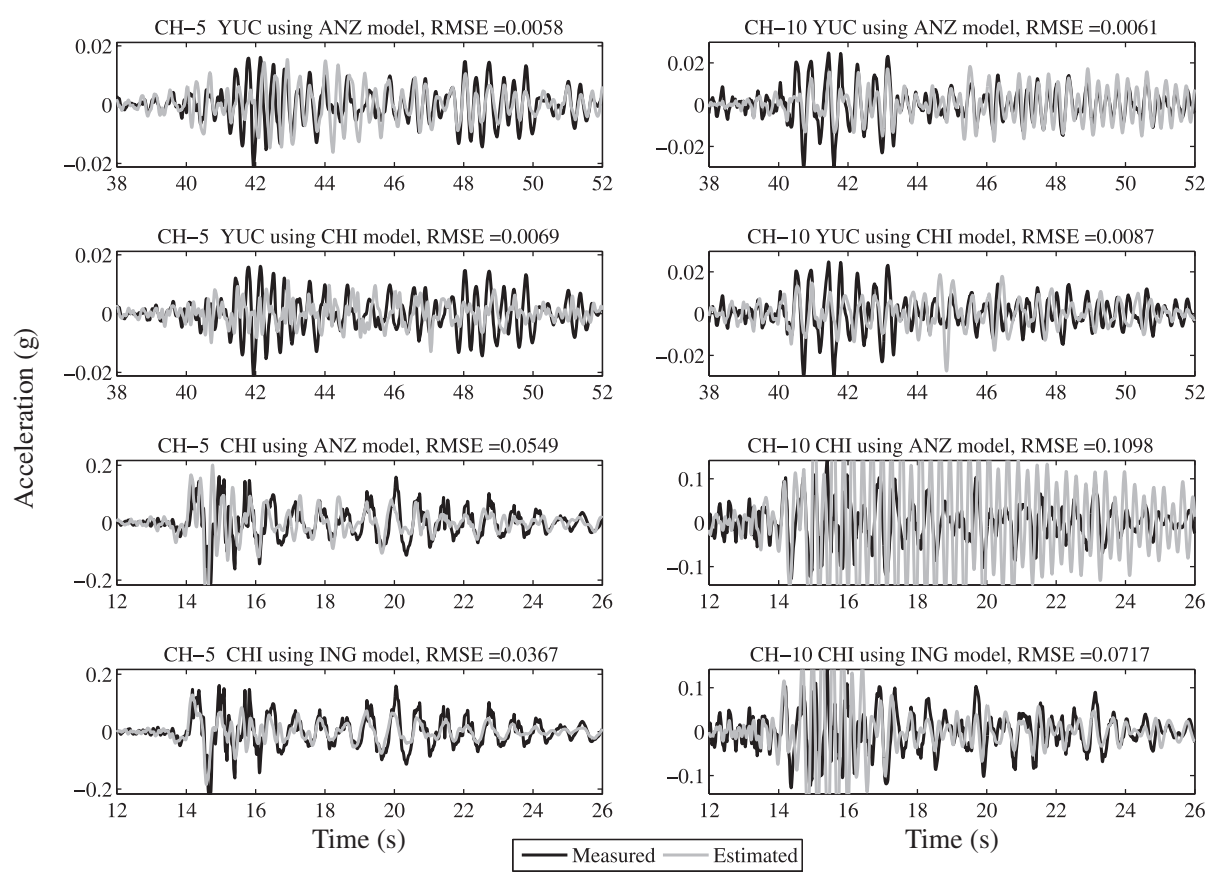

Figure 11. Predicted acceleration response at channels 5 (transverse) and 10 (vertical) using state space models constructed using MOESP technique. The predicted response is due to an earthquake different from that used to generate the model. 
using a state space model, each model is generated with a different earthquake recording. The models make an accurate prediction when the calculated response correlates to an earthquake of similar magnitude and intensity. In Figure 11 the response to the Yucaipa earthquake is predicted with less error using the model constructed using the Anza recordings $(\mathrm{RMSE}=0.0058)$. Similarly, adequate results were obtained in the prediction of the response to the Chino Hills earthquake when using the model constructed from the Inglewood recordings $(\mathrm{RMSE}=0.0367)$. However, the prediction of the response at channel 10 is poor and the models fail to predict the 'true-system-response', which is due to the lack of sufficient vertical response measurements. Despite the inaccuracies of the timehistory and disregarding the response at channel 10, it can be inferred that the peak acceleration for other channels is well predicted. Similar results were obtained using the ERA technique; however, RMSE values indicate that the MOESP technique gives slightly better results when predicting the bridge seismic response.

\section{DISCUSSION}

The three SI techniques utilized in this paper proved to be effective in identifying modal parameters and defining the state space models of a complex horizontally curved RC bridge from different seismic events even without preprocessing of the recorded data. However, only the first three natural frequencies of the bridge could be identified reliably. Higher modes were either inconsistent or not identified among different earthquakes and SI techniques. Also, the identified damping ratios were inconsistent between SI techniques, which revealed that identified damping values are not as reliable as natural frequencies and mode shapes. The accuracy of the identified damping ratios is difficult to evaluate and more data are needed to evaluate the accuracy of the results. In the future, more seismic records will enable a better estimation of the damping ratios.

Important observations about the seismic behavior of the WSOR can be mentioned such as the fact that the bridge tends to increase its flexibility when the earthquake intensity increases. On the basis of visual inspections, after an earthquake occurrence, it is unlikely that this structural softening was due to damage. Although imperceptible cracking of the columns is likely, this justifies the use of a reduced moment of inertia of the columns in design practice. However, as suggested by the sensitivity analysis, the explanation for an increase in the flexibility of the bridge is the change of the support conditions at both abutments and columns during ground shaking. Therefore, the transverse response of the WSOR during an earthquake is controlled mainly by the abutment motions.

In the case of the Chino Hills event it is interesting to observe that channel 5 located at the top of Bent 2 has a smaller peak acceleration $(0.22 g)$ than channel 7 located at the base of the same column $(0.37 \mathrm{~g})$. One possible explanation is that the response at the top of the piers depends on abutment restraining of the deck against torsion and lateral forces. If the restraining forces at the abutments are high, the pier top is prevented from displacing relative to its base. Indeed, if the abutments move during earthquakes, causing the opening of the bridge curvature in plan view, the pier top can potentially move opposite to the column base reducing the relative acceleration response between the base and top of Bent 2.

Usually, there exists an inherent variability of the natural frequencies because of environmental effects $[24,34]$. However, the approximately $20 \%$ change in the first natural frequency during the Chino Hills earthquake is significantly larger than any variation because of environmental effects, that is, wind, traffic, thermal effects. This can be corroborated from a previous study of the WSOR bridge where the change in the lower frequencies because of natural aging was evaluated. The change was found to be a consistent and gradual linear decrease of approximately $7 \%$ for the first natural frequency in a period of 8 years [26]. In that study regular traffic-induced vibration data including $10 \mathrm{~min}$ long ambient vibration records were utilized. Furthermore, during an earthquake, any change because of the aforementioned environmental effects have a very short time to act on the structural behavior and to be captured in a seismic record. For this reason the variation of the modal parameters found in this paper are attributed to the intensity of the earthquake. 


\section{CONCLUSIONS}

Identification of the modal parameters of WSOR bridge using six seismic records is presented in this paper. The results reveal that a significant reduction (nearly 20\%) of the lower natural frequencies of WSOR occur when the structure was subjected to the Chino Hills earthquake $\left(M_{\mathrm{w}}=5.5\right)$. Unfortunately, there are no sufficient seismic records of varying ground motion intensities to clarify whether the reduction in natural frequencies can be classified as a linear function of the earthquake intensity.

Identification results from a previous study were compared with the results obtained using the seismic records in this paper. In the previous study ambient and traffic induced vibration records were analyzed using an output-only SI technique. The comparison shows identification results before and after earthquake occurrence. It is concluded the variation of the bridge first natural frequency because of normal environmental effects is significantly smaller than the variation because of the action of moderate earthquakes such as the Chino Hills earthquake.

On the basis of visual inspections, the reduction in frequency during the earthquakes cannot be regarded as damage of the structural elements of the WSOR bridge. Sensitivity analysis shows that this variation is mainly due to the change of the support conditions because of softening of the soil surrounding the foundations during ground shaking.

Damping ratios tend to increase when the structure is subjected to earthquakes of larger magnitude and intensity. Typical values used in design practice (2\% to $7 \%$ of critical value) are obtained through SI. However, damping estimates are not as reliable as frequency estimates.

The first three identified mode shapes show a combination of horizontal and vertical components. This behavior is attributed to the curvature of the bridge and to the pronounced camber, having an inherent possibility of increasing the magnitude of the vertical response at the middle span.

The identified linear state space models gave a precise estimation of the acceleration response timehistory in the transverse direction. However, the match in vertical direction is not as reliable as the one in transverse direction because these linear models cannot simulate important effects produced by nonlinearities and interactions of the structure with surrounding soils and passing vehicles. Furthermore, it is the transverse motion that is significantly excited during ground shaking controlling the response of the structure.

The ability of the state space models to predict the response of a bridge structure to different earthquake events could be used for the assessment of the bridge dynamic behavior. Although the accuracy of the predictions could be low when matching measured time-history responses, it allows for a modest estimation of the peak acceleration at every sensor location on the bridge.

The presented approach focuses on the study of the variation of identified modal parameters from exclusively seismic records. The authors consider that this study contributes to the knowledge on highway bridges seismic behavior. However, there is still a need to evaluate the reasons causing the modal parameters to change because of a different earthquake intensity. Further research is needed to evaluate this phenomena and the lack of accuracy in the prediction of the vertical bridge seismic response using the state space models.

\section{ACKNOWLEDGEMENT}

The authors would like to acknowledge the support from the California Department of Transportation (contract number 59A0701), in particular to Mr. Li-Hong Sheng for his valuable comments on bridge maintenance, safety and management.

\section{REFERENCES}

1. Ulusoy HS, Feng MQ, Fanning PJ. System identification of a building from multiple seismic records. Earthquake Engineering and Structural Dynamics 2011; 40(6):661-674

2. Lus H, Betti R, Longman RW. Identification of linear structural systems using earthquake-induced vibration data. Earthquake Engineering and Structural Dynamics 1999; 28(11):1449-1467.

3. Lus H, Betti R, Longman RW. Obtaining refined first-order predictive models of linear structural systems. Earthquake Engineering and Structural Dynamics 2002; 31(7):1413-1440.

4. Ewins DJ. Modal Testing: Theory, Practice and Application. Research Studies Press: Baldock, Hertfordshire, U.K., 2000. 
5. Peeters B, De Roeck G. Stochastic system identification for operational modal analysis: a review. Journal of Dynamic Systems Measurement and Control-Transactions of the ASME 2001; 123(4):659-667.

6. Wilson JC. Analysis of the observed seismic response of a highway bridge. Earthquake Engineering and Structural Dynamics 1986; 14(3):339-354.

7. Werner SD, Beck JL, Levine MB. Seismic response evaluation of meloland road overpass using 1979 imperial-valley earthquake records. Earthquake Engineering and Structural Dynamics 1987; 15(2):249-274.

8. McCallen DB, Romstad KM. Dynamic analyses of a skewed short-span, box-girder overpass. Earthquake Spectra 1994; 10(4):729-755.

9. Desroches R, Fenves GL. Evaluation of recorded earthquake response of a curved highway bridge. Earthquake Spectra 1997; 13(3):363-386.

10. Loh CH, Lee ZK. Seismic monitoring of a bridge: Assessing dynamic characteristics from both weak and strong ground excitations. Earthquake Engineering and Structural Dynamics 1997; 26(2):269-288.

11. Arici Y, Mosalam KM. System identification of instrumented bridge systems. Earthquake Engineering and Structural Dynamics 2003; 32(7):999-1020.

12. Arici Y, Mosalam KM. Modal identification of bridge systems using state-space methods. Structural Control and Health Monitoring 2005; 12(3-4):381-404.

13. California Department of Transportation. 1983. Bridge Design Specifications, 1983 AASHTO with Interims and Revisions by Caltrans. Sacramento, California.

14. Arici Y, Mosalam KM. Modal Identification and Health Monitoring of Instrumented Bridges Using Seismic Acceleration Records. Technical Report, Earthquake Engineering Research Center, University of California, Berkeley. June 2006.

15. Aviram A, Mackie KR, Stojadinovic B. Effect of abutment modeling on the seismic response of bridge structures. Earthquake Engineering and Engineering Vibration 2008; 7(4):395-402.

16. Van Overschee P, De Moor B. N4SID-Subspace algorithms for the identification of combined deterministic stochastic-systems. Automatica 1994; 30(1):75-93.

17. Verhaegen M. Identification of the deterministic part of mimo state space models given in innovations form from input-output data. Automatica 1994; 30(1):61-74.

18. Larimore WE. Canonical variate analysis in identification, filtering, and adaptive-control. Proceedings of the 29th IEEE Conference on Decision and Control 1990; 2:596-604.

19. Viberg M. Subspace-based methods for the identification of linear time-invariant systems. Automatica 1995; 31(12): 1835-1851.

20. Van Overschee P, De Moor B. 1996. Subspace Identification for Linear Systems: Theory, Implementation, Applications. Kluwer Academic Publishers: Dordrecht, Netherlands.

21. Juang JN, Pappa RS. An Eigensystem Realization Algorithm for Modal Parameter Identification and Model reduction. Journal of Guidance, Control, and Dynamics 1985; 8(5):620-627.

22. Juang JN, Phan M, Horta LG, Longman RW. Identification of Observer Kalman/Filter Markov parameters - theory and experiments. Journal of Guidance, Control, and Dynamics 1993; 16(2):320-329.

23. Ho BL, Kalman RE. Effective construction of linear state-variable models from input/output data. Proceedings of the 3rd Annual Allerton Conference on Circuit and System Theory 1965; 449-459.

24. Peeters B, De Roeck G. One-year monitoring of the Z24-Bridge: environmental effects versus damage events. Earthquake Engineering and Structural Dynamics 2001; 30(2):149-171.

25. Van der Auweraer H, Peeters B. Discriminating physical poles from mathematical poles in high order systems: use and automation of the stabilization diagram. Proceedings of the 21st IEEE, Instrumentation and Measurement Technology Conference, IMTC 2004; 3:2193-2198.

26. Gomez HC, Fanning PJ, Feng MQ. Testing and long-term monitoring of a curved concrete box girder bridge. Engineering Structures 2011; 33(10):2861-2869.

27. Farrar CR, James GH. System identification from ambient vibration measurements on a bridge. Journal of Sound and Vibration 1997; 205(1):1-18.

28. Alvin KF, Robertson AN, Reich GW, Park KC. Structural system identification: from reality to models. Computers and Structures 2003; 81(12):1149-1176.

29. Lee S, Feng MQ, Kwon SJ, Hong SH. Equivalent Modal Damping of Short-Span Bridges Subjected to Strong Motion. Journal of Bridge Engineering-ASCE 2011; 16(2):316-323.

30. Mitchell L. Complex modes: a review. Proceedings of the 8th International Modal Analysis Conference, IMAC Kissimmee, FL, 1990; 2:891-899.

31. Niedbal N. Analytical determination of real normal modes from measured complex responses. Proceedings of the 25th Structures, Structural Dynamics and Materials Conference Palm Springs, CA, 1984; 292-295.

32. Allemang RJ, Brown DL. A correlation coefficient for modal vector analysis. Proceedings of the 1st International Modal Analysis Conferencce, IMAC Orlando, FL, 1982; 110-116.

33. Richardson JA, Douglas BM. Results from field testing a curved box-girder bridge using simulated earthquake loads. Earthquake Engineering and Structural Dynamics 1993; 22(10):905-922.

34. Farrar C, Doebling SW, Cornwell PJ, Straser EG. Variability of modal parameters measured on the Alamosa Canyon Bridge. Proceedings of the 15th International Modal Analysis Conference, IMAC Orlando, FL, 1997; 257-263. 